\title{
Quantifying the Aggregation Factor in Carbon Nanotube Dispersions by Absorption Spectroscopy
}

\author{
Hari Pathangi, ${ }^{1}$ Philippe M. Vereecken, ${ }^{2}$ Alexander Klekachev, ${ }^{3}$ \\ Guido Groeseneken, ${ }^{4}$ and Ann Witvrouw ${ }^{3}$ \\ ${ }^{1}$ IMEC, Kapeldreef 75, B3001 Leuven, Belgium \\ ${ }^{2}$ Centre for Surface Chemistry and Catalysis, KU Leuven, B3001 Leuven, Belgium \\ ${ }^{3}$ Department of Physics, KU Leuven, B3001 Leuven, Belgium \\ ${ }^{4}$ Department of Electrical Engineering, KU Leuven, B3001 Leuven, Belgium
}

Correspondence should be addressed to Hari Pathangi; pathangi@imec.be

Received 16 December 2013; Revised 15 March 2014; Accepted 1 April 2014; Published 29 April 2014

Academic Editor: Ana Benito

Copyright (C) 2014 Hari Pathangi et al. This is an open access article distributed under the Creative Commons Attribution License, which permits unrestricted use, distribution, and reproduction in any medium, provided the original work is properly cited.

\begin{abstract}
Absorption spectroscopy in the ultraviolet-visible-near infrared (UV-Vis-NIR) wavelength region has been used to quantify the aggregation factor of single-walled carbon nanotubes (SWCNTs) in liquid media through a series of controlled experiments. SWCNT bundles are dispersed in selected solvents using a calibrated ultrasonicator, which helps in determining the true amount of energy used in the exfoliation process. We also establish the selectivity of the centrifugation process, under the conditions used, in removing the nanotube aggregates as a function of the sonication time and the dispersion solvent. This study, along with the calibration of the sonication process, is shown to be very important for measuring the true aggregation factor of SWCNTs through a modified approach. We also show that the systematic characterization of SWCNT dispersions by optical spectroscopy significantly contributes to the success of dielectrophoresis (DEP) of nanotubes at predefined on-chip positions. The presence of individually dispersed SWCNTs in the dispersions is substantiated by dielectrophoretic assembly and post-DEP electromechanical measurements.
\end{abstract}

\section{Introduction}

Single-walled carbon nanotubes (SWCNTs) have attracted significant interest in basic and applied nanomaterials research $[1,2]$ due to their exceptional electrical [3], mechanical [4], optical [5], and thermal properties [6]. In order to exploit these attractive properties [7], SWCNTs have been proposed as components in a variety of applications like sensors [8,9], field effect transistors [10], interconnects in CMOS technology [11], electromechanical springs [12], and field emission sources [13], as additives in composite materials for enhanced mechanical properties [14], and as medical therapeutic agents [15]. In spite of their huge promise, the success of SWCNT devices still remains uncertain at a commercial level. This is because SWCNTs exist in a wide range of diameters, lengths, chiralities (the rollup axis), structural purity, and states of aggregation [16]. Therefore, fabrication schemes need good selectivity in order to control the physical properties of SWCNTs and thus their device properties. This selectivity can be obtained either through controlled growth to limit the variability among the as-grown nanotubes [17-19] or postgrowth purification and sorting techniques [20-22].

SWCNTs in the native form exist in a bundled state. For a number of the postgrowth SWCNT sorting techniques, it is desired to have the nanotubes dispersed uniformly in a liquid medium [20-22]. It is, however, difficult to disperse them efficiently in water and most other common solvents [23]. This is mainly because of the high intertube van der Waals force of attraction [23], which has to be overcome to separate the nanotube bundles and suspend them in a solvent. The choice of a suitable solvent is therefore essential. There have been many efforts in the recent past to efficiently exfoliate SWCNTs in water, with the help of surfactants $[24,25]$ or sidewall functionalization [26], and in organic solvents [27, 28]. For most applications the surfactant residues must be removed from the nanotubes and/or the substrate after processing to avoid 
undesired effects of the surfactant molecules on the SWCNT properties. It is therefore preferable to disperse the SWCNTs in a two-component system (nanotubes-solvent) rather than a three-component system (nanotubes-surfactants-solvent). Removal of residual organic solvent from the nanotubes could be achieved by relatively straightforward techniques like rinsing and/or thermal treatment. This is expected to be easier compared to the removal of surfactant residues, which might interact chemically with the nanotubes [28]. Hence, a solvent having a true thermodynamic solubility for the nanotubes would be an elegant solution [29-31].

An ideal dispersion solvent is one in which SWCNTs can be uniformly dispersed over a broad concentration range with minimal levels of aggregation. It is also preferred that the resulting dispersions are stable over time without chemical or structural modifications to the nanotubes [32]. Recently, a number of amide based solvents $[32,33]$ have been successfully used as effective media to prepare SWCNT dispersions, specifically N-methyl pyrrolidone (NMP) and its chemical analogues [31]. The exact mechanism of the SWCNT dispersion process is yet to be established [34]. There are, however, indications that NMP and other amide-based solvents might result in strong nanotube-solvent interactions. The surface energy of these solvents is of the order of $40-$ $50 \mathrm{~mJ} \mathrm{~m}^{-2}$ [31], which is comparable to that of nanotubes (70$80 \mathrm{~mJ} \mathrm{~m}^{-2}$ ) [31]. This results in a low energy barrier for the exfoliation-dispersion process [30].

Uniform aggregates-free SWCNT dispersions have been successfully prepared in different amide-based solvents with a combination of sonication and centrifugation processes [26]. Sonication (SO) accelerates the rate of exfoliation of the nanotubes in the dispersion solvent. However, care should be taken not to induce damage to the nanotubes by extensive sonication. Therefore, a centrifugation (CF) step is often used after mild sonication to remove the undispersed SWCNT aggregates, while leaving behind the dispersed nanotubes, which could be either individually dispersed or nanotubes in the form of narrow bundles [26]. The efficiency of the sonication process is related to the net sonication energy input, which is determined by the output power of the sonicator, frequency, and time. For a direct comparison of the conditions published across the various reports, the net sonication energy input should be known in addition to the sonication time, as rightly pointed out by Bergin et al. [31].

In the literature $[35,36]$, ultraviolet-visible-near infrared spectroscopy (UV-Vis-NIR) has been widely used to determine the level of aggregation of SWCNTs in liquid dispersions. Also, SWCNT purity, diameter, length, and chirality have been determined from spectroscopic studies, often in combination with other techniques such as scanning electron microscopy (SEM), transmission electron microscopy (TEM), thermogravimetric analysis (TGA), and Raman spectroscopy [35]. Atomic force microscopy (AFM) has often been used to study the diameter distribution of the nanotubes and their level of aggregation after drop-casting or spincoating them onto a substrate $[32,33]$. The atomic resolution of the AFM is useful to precisely measure the diameter of individual nanotubes. However, AFM might not be the most suitable technique to determine the aggregation level of nanotubes spin-coated on a substrate from a liquid dispersion. This is because the spin-coating process itself might induce additional aggregation of nanotubes on the substrate especially during the drying of the dispersion solvent. Therefore, characterizing the level of nanotube aggregation in the liquidphase (and not on a substrate) using spectroscopic techniques might be more precise and appropriate for practical applications.

For the fabrication of SWCNT devices using liquidphase self-assembly techniques, the characterization of the nanotubes and their level of aggregation in the dispersionphase is particularly important. We report a characterization scheme to quantify the aggregation factor of SWCNTs in liquid dispersions using optical spectroscopy after a controlled sonication-centrifugation process. We studied the true selectivity of the centrifugation process in removing the SWCNT aggregates with respect to the dispersed nanotubes. This has led to a modified approach to calculate the aggregation factor of SWCNTs in liquid dispersions. For our study, we have chosen NMP, a solvent which is considered effective for SWCNT dispersion [31-33], and isopropyl alcohol (IPA), a solvent which is considered ineffective for SWCNT dispersion [27]. An important step in our approach is the calibration of the ultrasonication in terms of the true energy input, which helps in standardizing the sonication process. Electron microscopy after directed assembly of the SWCNTs by dielectrophoresis (DEP) and post-DEP electromechanical measurements has been used to substantiate the presence of individually dispersed nanotubes in the dispersions. The dielectrophoresis experiments also confirmed the effect of centrifugation in removing SWCNT aggregates from the liquid dispersions, increasing the fraction of individual nanotubes. A number of reports have been published dealing with the characterization of SWCNT aggregation by absorption spectroscopy [32-40]. The novelty of our work lies in the manner in which we demonstrate a direct relevance of our spectroscopic studies to successfully achieve controlled SWCNT assembly by dielectrophoresis. This is important for practical applications, as techniques like dielectrophoresis can be used to fabricate arrays of SWCNT devices in parallel with the desired nanotube density and orientation at predefined on-chip locations.

\section{Materials and Methods}

Ultrapure SWCNT samples synthesized by arc discharge process were purchased from NanoIntegris in the form of a SWCNT thin film (product name: super-pure tubes: batch no. PC10-478) with metal catalyst impurity $<1 \%$ and amorphous carbon impurity $<5 \%$. The nanotubes, according to the manufacturer's technical data sheet, were $300 \mathrm{~nm}$ to $4 \mu \mathrm{m}$ long (mean: $2 \mu \mathrm{m}$ ) and had an average diameter of $1.5 \mathrm{~nm}$. The nanotube dispersions were prepared with NMP (Merck, 99.5\%) and IPA (Honeywell, ULSI Puranal) as solvents. SWCNT samples were weighed in an AllScales Europe mass balance (rated between $0.1 \mathrm{mg}$ and $120 \mathrm{~g}$ ) and dispersed in the solvent using a Hielscher ultrasonicator (UP200S). 


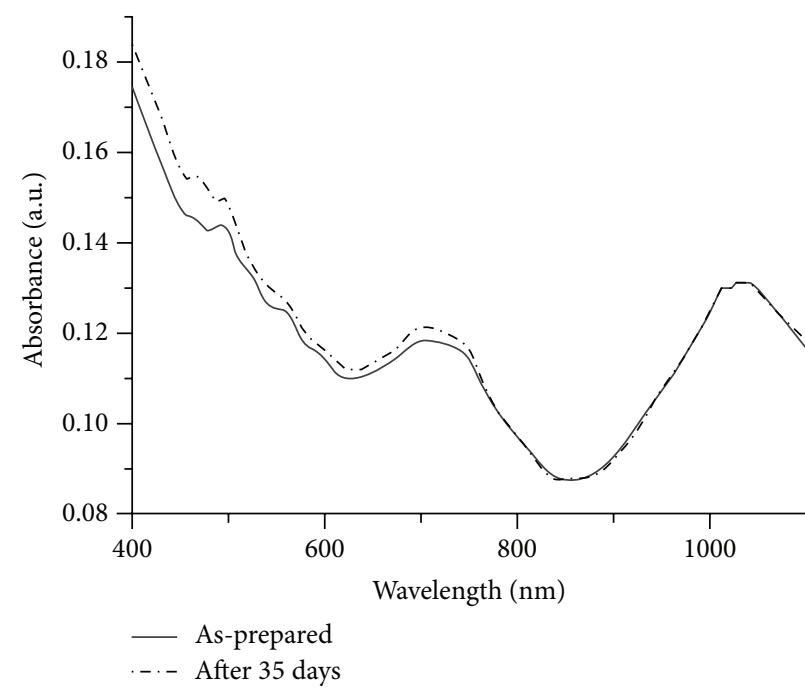

(a)

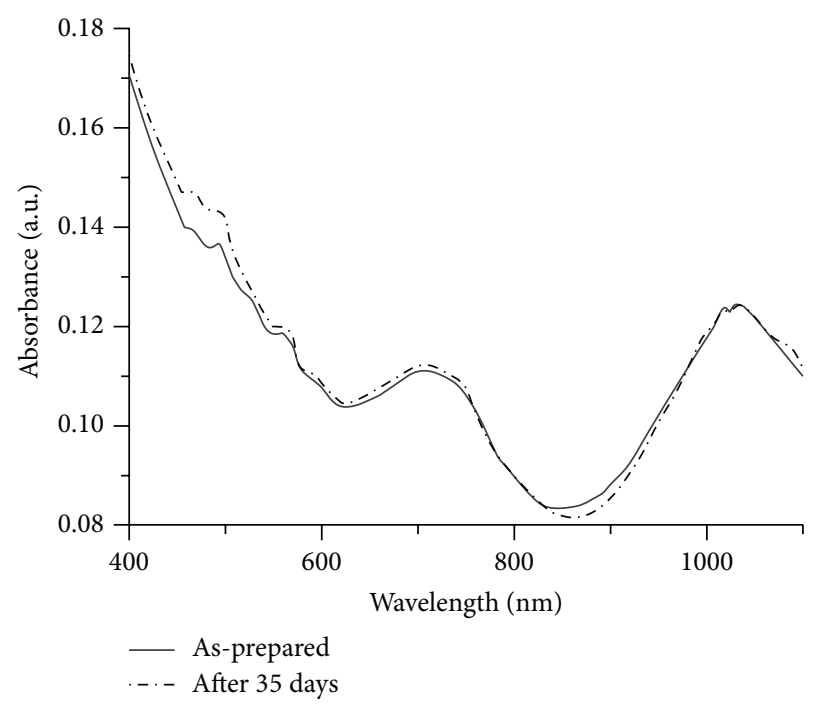

(b)

FIGURE 1: Optical absorption spectra of $2 \mu \mathrm{g} / \mathrm{mL}$ SWCNT dispersions in NMP (a) after 20 minutes sonication only and (b) after 20 minutes sonication followed by centrifugation. The as-prepared spectra (solid curves) were measured immediately after the dispersions were prepared and the after 35 days spectra (dashed-dotted curves) were measured for the same dispersion 35 days after it was prepared with (a) sonication only or (b) sonication and centrifugation. The absorbance of the dispersions was measured with respect to the blank solvent in absorbance units (a.u.).

The ultrasonicator was rated for a maximum power output of $200 \mathrm{~W}$ in aqueous media at a working frequency of $24 \mathrm{kHz}$. The dispersions were sonicated for different durations with the Tip S7 titanium sonotrode (7 mm tip diameter) at 50\% power output. For studying the dispersion stability over time (Figure 1), $2 \mu \mathrm{g} \mathrm{mL}^{-1}$ dispersions in NMP were prepared by sonicating for 20 minutes. Centrifugation was carried out with a $10 \mathrm{~mL}$ sample of each dispersion (in $14 \mathrm{~mL}$ BD falcon tubes) using a Sigma Laboratory Centrifuge (2K15) at a rotational speed corresponding to $8500 \mathrm{~g}$ (8945 rpm) for 90 minutes. Optical spectra of the SWCNT dispersions were recorded in a Shimadzu (UV-1601PC) spectrophotometer versus blank NMP or IPA solvents. Plastic cuvettes (Eppendorf UVette) with an optical path length of $10 \mathrm{~mm}$ were used for the absorbance measurement. The random error in the optical absorbance measurement (0.0024 a.u.) was determined by measuring the absorbance of the blank NMP solvent at a fixed wavelength of $1030 \mathrm{~nm}$ with respect to time (for 2 hours). To study the Beer's law dependence (Figure 2), $60 \mu \mathrm{g} \mathrm{mL}^{-1}$ dispersions in NMP and IPA were freshly prepared as the parent dispersion with 20 minutes of sonication. The $60 \mu \mathrm{g} \mathrm{mL}^{-1}$ dispersion was then successively diluted with NMP or IPA to obtain the $30,15,7.5$, 3.8, and $1.9 \mu \mathrm{g} \mathrm{mL}^{-1}$ dispersions and each dispersion was then sonicated for an additional 30 seconds to homogenize. Centrifugation was always carried out immediately after the sonication of each dispersion. The centrifugation parameters remained constant for all the dispersions across the various studies. Only the sonication time (energy input) was varied. The optical spectra reported in Figure 2 were measured immediately after sonication or sonication-centrifugation. For the experiment to study the effect of sonication time on the apparent concentration (Figure 6), a series of $7.5 \mu \mathrm{g} \mathrm{mL}^{-1}$ dispersions in NMP was freshly prepared and optical spectra were measured after the different sonication durations (with or without centrifugation).

The presence of individually dispersed SWCNTs was substantiated by dielectrophoretic assembly of SWCNTs from the NMP dispersions onto electrode patterns. Arrays of triangular electrode pairs were fabricated on a $200 \mathrm{~mm}$ silicon wafer with a $500 \mathrm{~nm}$ thick thermal oxide on top. The $150 \mathrm{~nm}$ thick titanium nitride (TiN) electrode pairs were fabricated using deep-UV lithography and dry etch of TiN. A series of SWCNT dispersions in NMP was prepared with the following concentrations: $1 \mu \mathrm{g} / \mathrm{mL}, 500 \mathrm{ng} / \mathrm{mL}$, and $250 \mathrm{ng} / \mathrm{mL}$. The dispersions were sonicated for 20 minutes (sonication energy input of $16 \mathrm{~kJ}$ ) and a $10 \mathrm{~mL}$ sample from each dispersion was centrifuged at the above mentioned conditions. The sonicated dispersions before and after centrifugation were each used in different DEP experiments. Dielectrophoretic assembly was carried out on $2 \times 2 \mathrm{~cm}^{2}$ sized dies containing the electrode pairs on a 4-inch wafer prober in the presence of an applied electric field. A combination of $2 \mathrm{~V}$ DC bias and an $\mathrm{AC}$ voltage with $8 \mathrm{~V}(\mathrm{p}-\mathrm{p})$ at $2 \mathrm{MHz}$ frequency was applied on the electrodes, while applying a $35 \mu \mathrm{L}$ droplet of the dispersion for 5 seconds on the die. The die was then rinsed with water and ethanol and dried using a critical point dryer, to avoid stiction in nanotubes due to the capillary forces. A Nova200 NanoSEM electron microscope (FEI) was used to image the electrode structures after SWCNT assembly with $6.5 \mathrm{keV}$ beam acceleration voltage at $4 \mathrm{~mm}$ working distance. The suspended nanotubes assembled between the electrode pairs were then subjected to electromechanical measurements. Measurements were carried out in a probe 


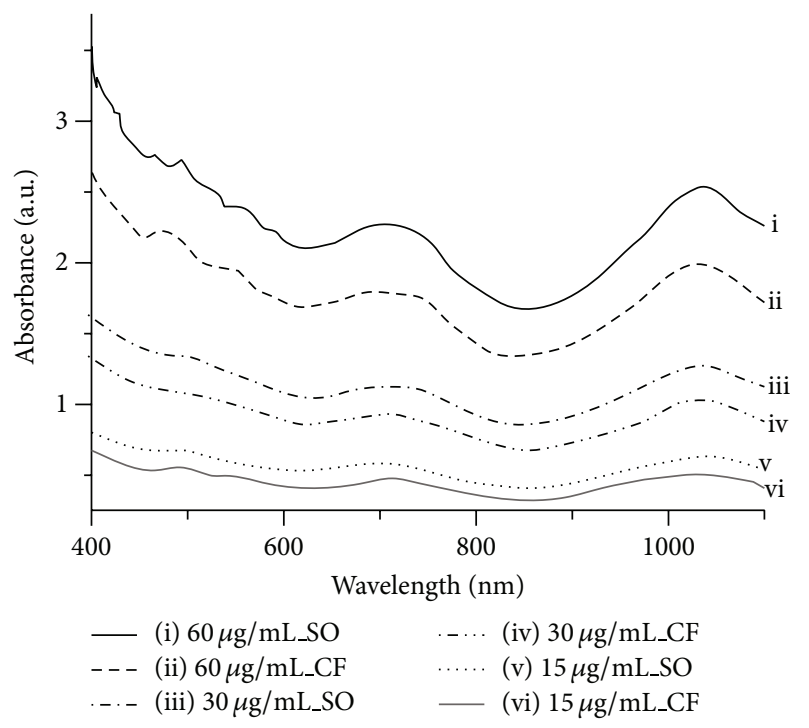

(a)

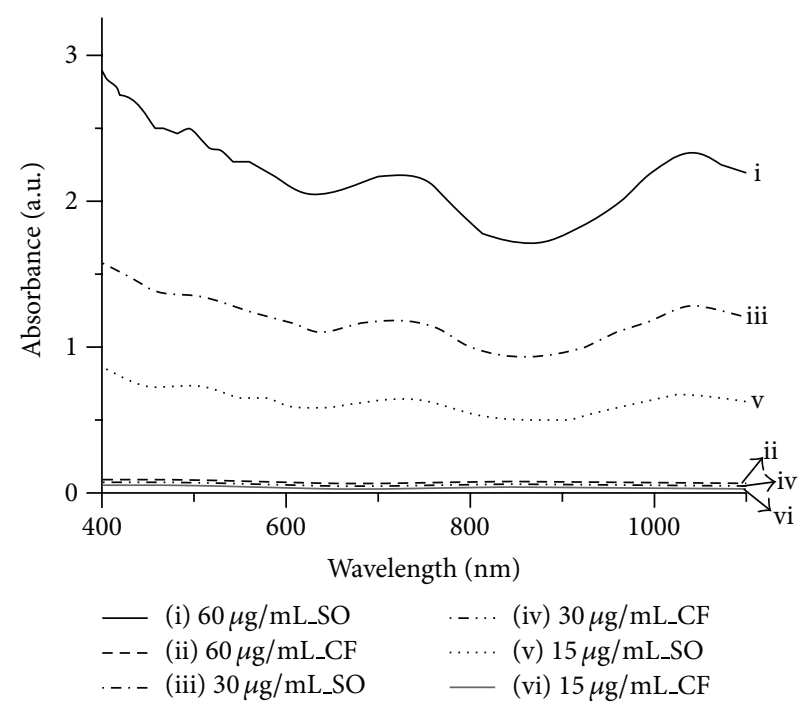

(b)

Figure 2: Absorbance spectra of SWCNT dispersions in (a) NMP and (b) IPA for $60 \mu \mathrm{g} / \mathrm{mL}$ ((i) and (ii)), $30 \mu \mathrm{g} / \mathrm{mL}$ ((iii) and (iv)), and $15 \mu \mathrm{g} / \mathrm{mL}((\mathrm{v})$ and (vi)) after 20 minutes sonication only ((i), (iii), and (v)) and after 20 minutes sonication followed by centrifugation ((ii), (iv), and (vi)).

station with a vacuum chamber (Janis Research Company) at room temperature and $6 \times 10^{-4} \mathrm{mbar}(0.06 \mathrm{~Pa})$ pressure. A Keithley 220 programmable current source was used to drive a constant current through the nanotube between the two electrodes. The sample was mounted on a copper plate $\left(2 \times 2 \mathrm{~cm}^{2}\right)$ with a dielectric spacer at the bottom to decouple the $\mathrm{p}^{++}$doped Si substrate from the sample holder of the chamber. The Si substrate was used as a back-gate to which a DC voltage (Agilent E3647A power supply) with superimposed AC signal (Rohde and Schwarz SMBV 100A vector signal generator) was applied with the help of a bias$\mathrm{T}$. The frequency of the AC signal applied at the substrate for actuation was swept from $10 \mathrm{kHz}$ to $50 \mathrm{MHz}$. The DC voltage drop across the two electrodes bridged by the nanotube was monitored, using a Keithley digital multimeter, as a function of the AC signal frequency. The resistance of the nanotube was calculated from the voltage drop, normalized, and then plotted as a function of the actuation frequency.

\section{Results and Discussion}

3.1. Effective Sonication Energy Input. Ultrasonication, widely used for SWCNT exfoliation in solvent media, is known to induce mechanical damage to the nanotubes $[28,32,35$, 38]. To ensure adequate nanotube quality in the resulting dispersion, it is important to quantify and control the sonication energy (SE) that is supplied to the system. The net $\mathrm{SE}$ in our experiments was determined by measuring the temperature rise $(\Delta T)$ of a known mass of blank NMP solvent $(m)$ after sonication for different durations. The maximum temperature reached during this sonication was $63^{\circ} \mathrm{C}$. As NMP has a boiling point of $204^{\circ} \mathrm{C}$ and a low vapor pressure of $0.3-0.5 \mathrm{~mm} \mathrm{Hg}$, we could safely disregard the effects of evaporation during these experiments. From the known specific heat capacity of NMP $\left(C=1.95 \mathrm{Jg}^{-1} \mathrm{~K}^{-1}\right)$, the amount of energy provided to the system $(Q)$ was calculated from the equation $Q=C \cdot m \cdot \Delta T$. The SE per unit time (true output power) was then calculated to be $14 \mathrm{~W}$ for the tip sonicator operated at $50 \%$ output power (calibrated at $200 \mathrm{~W}$ total output power) with continuous sonication.

3.2. SWCNT Quality. Raman spectroscopy was used to determine the effect of sonication energy input on the quality of the nanotubes. Figure 12(a) shows the Raman spectrum of the as-purchased nanotube sample with the radial breathing mode (RBM) at $164 \mathrm{~cm}^{-1}$, a signature for the presence of SWCNTs. For the as-received SWCNT sample, the G peak corresponding to the in-plane stretching of the carbon atoms in the graphite lattice can be observed at $1590 \mathrm{~cm}^{-1}$ and the $\mathrm{D}$ peak (originating from the structural defects of the nanotubes) is at $1322 \mathrm{~cm}^{-1}$ (Figure 12(a)).

The ratio of intensities of the $G$ and $D$ peaks in the SWCNT spectrum $\left(I_{\mathrm{G}} / I_{\mathrm{D}}\right)$ is a measure for the nanotube quality [35]. A higher $I_{\mathrm{G}} / I_{\mathrm{D}}$ ratio implies a better structural quality of the nanotubes. The unprocessed SWCNT sample exhibits $I_{\mathrm{G}} / I_{\mathrm{D}}$ value of 15 , while it decreases to 5 after 30 minutes of sonication (corresponding to $25 \mathrm{~kJ}$ of sonication energy input). Figure 12 (c) shows a steady decrease in $I_{\mathrm{G}} / I_{\mathrm{D}}$ as a function of increasing sonication energy input, due to the damage of the SWCNTs induced by sonication.

\subsection{UV-Vis-NIR Characterization}

3.3.1. Stability of NMP Dispersions. First, the stability of the SWCNT dispersion in NMP was checked with optical absorption measurements. Figure 1 shows the optical 
spectra of $2 \mu \mathrm{g} / \mathrm{mL}$ SWCNT dispersion in NMP measured immediately upon preparation and 35 days later. Due to the difference in the energy of their electronic transitions, metallic and semiconducting nanotubes result in distinct optical absorption peaks [36]. The two main features in the absorbance spectra corresponding to the optical transitions of the metallic and the semiconducting SWCNTs, namely, the M11 and S22 transitions [36], are, respectively, centered around $750 \mathrm{~nm}$ and $1030 \mathrm{~nm}$ (NanoIntegris: technical data sheet for unsorted super-pure tubes prepared by arcdischarge method).

The absorption spectra measured immediately and 35 days after preparation were comparable, indicating that the dispersions were stable over time and that there is no considerable reaggregation over this time period. Also, no appreciable difference between the absorbance maxima at $1030 \mathrm{~nm}\left(A_{1030}\right)$ was observed for the dispersions without (0.13 a.u.; Figure 1(a)) and with centrifugation ( 0.12 a.u; Figure 1(b)). This can be attributed to the rather low initial concentration of the dispersion $(2 \mu \mathrm{g} / \mathrm{mL})$ and the long sonication duration of 20 minutes, which result in almost complete dispersion of the SWCNTs. A similar trend was also observed for dispersions (of the same concentration) sonicated for different durations. Detailed studies of the effects of sonication time and concentration on the centrifugation process will be presented in the following sections.

3.3.2. Absorbance versus Concentration. Two series of SWCNT dispersions with concentrations between 1.9 and $60 \mu \mathrm{g} \mathrm{mL}^{-1}$ were prepared, one with NMP and another with IPA as the solvent. Figure 2 shows some typical optical absorption spectra for dispersions of various concentrations after sonication only and after sonication and centrifugation.

For the dispersions with sonication only, the magnitude of $A_{1030}$ decreases with the as-prepared concentration, as expected from Beer's law. After centrifugation, the peak intensities did not change much for the dispersions in NMP. For the dispersions in IPA, on the other hand, the peak intensities dropped drastically after centrifugation. The absorbance maxima at $1030 \mathrm{~nm}\left(A_{1030}\right)$ and at $660 \mathrm{~nm}\left(A_{660}\right)$ are plotted in Figures 3(a) and 3(b), respectively, as a function of the as-prepared concentration.

The absorbance for both the NMP and the IPA dispersions measured immediately after sonication exhibits a good proportionality according to Beer's law $\left(A_{\lambda}=\varepsilon_{\lambda} c l\right) ; A_{\lambda}$ is the measured absorbance at a particular wavelength $(\lambda)$, $\varepsilon_{\lambda}$ is the molecular extinction coefficient at $\lambda[31], c$ is the concentration of the dispersion, and $l$ is the optical path length in the spectrophotometer. This generally indicates the presence of well dispersed nanotubes in the dispersions measured immediately after sonication. Beer's law is a simple empirical law describing the relation between the absorbance and the concentration for dilute solutions. The molecular extinction coefficient at $1030 \mathrm{~nm}\left(\varepsilon_{1030}\right)$ was therefore calculated from the slope in Figure 3(c) for $A_{1030}<1$ a.u. and was calculated to be $4690 \mathrm{~mL} \mathrm{mg}^{-1} \mathrm{~m}^{-1}$. The molecular extinction coefficient at $660 \mathrm{~nm}\left(\varepsilon_{660}\right)$ was calculated to be
$3600 \mathrm{~mL} \mathrm{mg}^{-1} \mathrm{~m}^{-1}$. This value for our arc-discharge nanotubes is in reasonable agreement with the results obtained by Landi et al. [34] and Bergin et al. [31], who reported $\varepsilon_{660}$ values between 3000 and $3470 \mathrm{~mL} \mathrm{mg}^{-1} \mathrm{~m}^{-1}$ for purified nanotubes produced by the HiPco (high pressure carbon monoxide) process. For the subsequent discussions, we will use the extinction coefficient at $1030 \mathrm{~nm}\left(\varepsilon_{1030}\right)$ for calculating the effective concentration of the SWCNT dispersions. It is worth noting that the dispersed SWCNTs present in the dispersions after 20 minutes of sonication could still be either individually dispersed nanotubes or present in the form of narrow bundles. This will be clarified in the forthcoming sections.

The absorbance spectra obtained from the NMP and the IPA dispersions after sonication are similar to the function of the as-prepared concentration (Figure 3), when measured immediately after sonication. In the case of IPA, a small deviation from the linear fit was observed for concentrations larger than $30 \mu \mathrm{g} / \mathrm{mL}$, indicating some level of aggregation. It is worth noting that in addition to the individually dispersed nanotubes, the SWCNT aggregates contribute to the absorbance measurements. The nanotube clusters present in the dispersion lower the effective absorbance measured due to shielding effects of the bundles [37]. The similar trend for IPA and NMP after sonication is not, however, truly representative of the effectiveness of the two solvents for dispersing SWCNTs. Indeed, a drastic drop in the absorbance is seen for the dispersions in IPA measured 90 minutes after sonication. This suggests a much higher tendency of the SWCNTs to reaggregate in IPA, compared to the dispersions in NMP, which remained stable over a period of at least 35 days (Figure 1). Figure 2(b) also revealed a drastic drop in the absorbance intensities of the dispersions in IPA after centrifugation. This suggests that centrifugation itself may have induced or accelerated the reaggregation of SWCNTs in IPA. For all the above experiments, the dispersions were sonicated for 20 minutes and the centrifugation was carried immediately afterwards. In practice, such long sonication durations to effectively disperse the nanotubes cannot always be used, as this increases the possibility of inducing structural damage to the nanotubes (refer to the supplementary data for Raman spectra). Centrifugation is therefore often used to remove the nanotube aggregates that were not dispersed after mild sonication. The following sections will elaborate on the true selectivity of the centrifugation process, which further confirms the difference between NMP and IPA for effectively dispersing nanotubes.

\subsection{SWCNT Aggregation}

3.4.1. Effect of Centrifugation. There have been numerous reports [31-40] to quantify the fraction of aggregated nanotubes $(\chi)$ using optical absorption spectroscopy by studying the absorbance before $\left(A_{\lambda, i}\right)$ and after $\left(A_{\lambda, f}\right)$ centrifugation of sonicated SWCNT dispersions. Consider

$$
\chi=\frac{A_{\lambda, i}-A_{\lambda, f}}{A_{\lambda, i}} .
$$




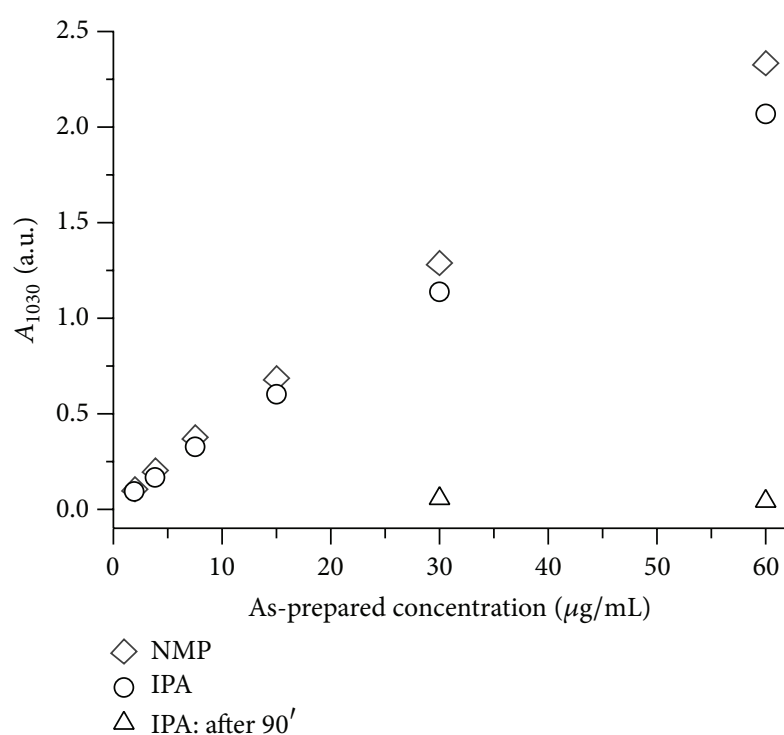

(a)

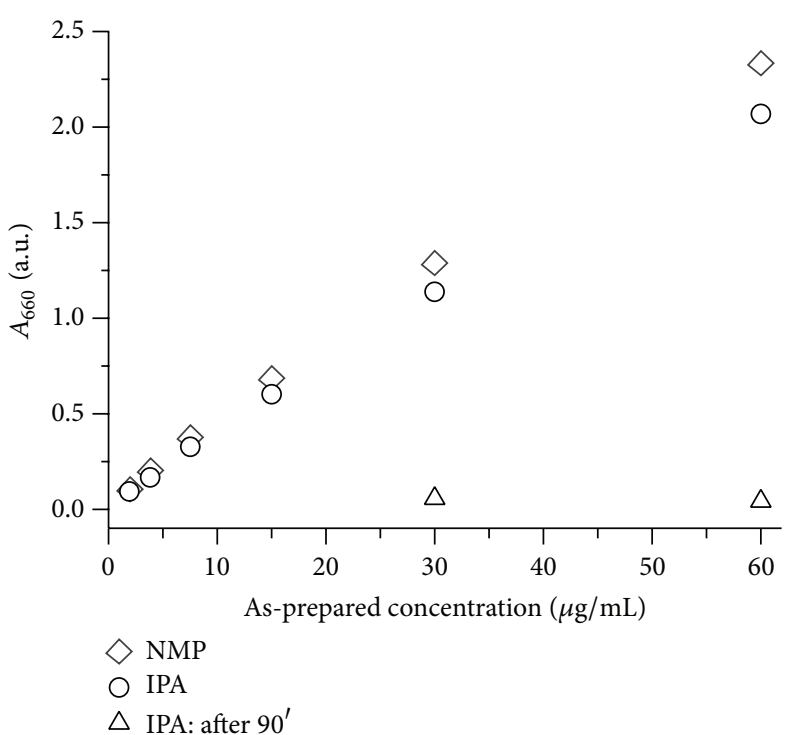

(b)

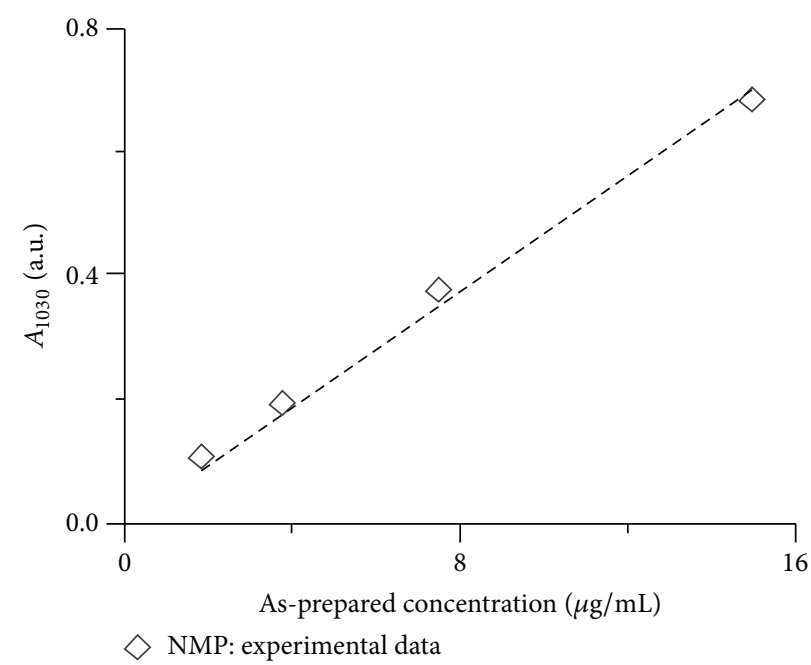

(c)

Figure 3: Absorbance of the NMP dispersions and the IPA dispersions versus the as-prepared concentration at (a) $1030 \mathrm{~nm}, A_{1030}$ and (b) $660 \mathrm{~nm}, A_{660}$. The dispersions ( $200 \mathrm{~mL}$ each) were sonicated for 20 minutes (energy input of $0.08 \mathrm{KJ} / \mathrm{mL}$ ) and the spectra for NMP and IPA were measured immediately after sonication. The absorbance of the dispersions in IPA was remeasured 90 minutes after sonication. (c) The molecular extinction coefficient, $\varepsilon_{1030}$, was calculated from the slope of the linear fit (dashed line) to the measurements with the dispersions in NMP using the absorbance values less than 1 a.u.

Equation (1) assumes that centrifugation removes the nanotube bundles, while the dispersed tubes are left suspended in the solvent. However, the true selectivity of centrifugation in removing the SWCNT aggregates with respect to the dispersed nanotubes needs to be checked first. Therefore, optical spectra of sonicated NMP and IPA dispersions of various concentrations were measured before and after centrifugation (Figure 2). It has been established in the literature that the molecular extinction coefficient being a material property is not dependent on the level of aggregation of the SWCNTs in the dispersion $[31,34]$. Also, the effect of shielding of aggregates on the measured absorbance value can be neglected after centrifugation as the number of aggregates is minimal (will be confirmed in the following section). Hence, knowing $\varepsilon_{1030}$ (calculated from Figure 3(a)) and from the absorption data in Figure 2, we can estimate the effective concentration of the various dispersions after centrifugation. The difference in the change in the SWCNT concentration after centrifugation for the NMP and IPA dispersions is shown in Figure 4.

In the case of IPA, centrifugation removed about 7095\% of the nanotubes from the dispersion, which is expected as IPA is known to be an ineffective solvent for dispersing SWCNTs. However, $14-21 \%$ of the nanotubes were still removed from the dispersions in NMP (considered to be a good dispersion solvent for SWCNTs) even after 20 minutes 


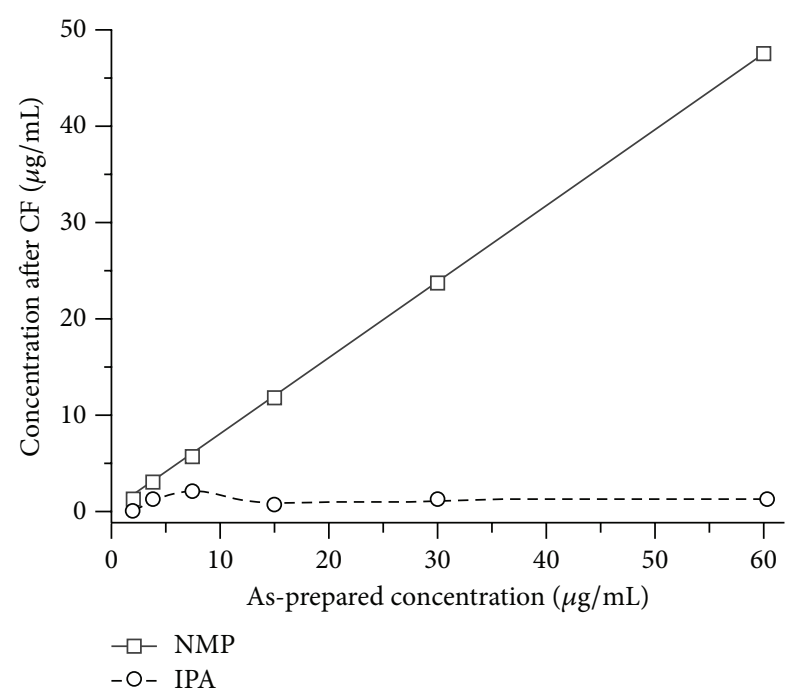

FIGURE 4: Relationship between the SWCNT concentration after sonication-centrifugation (determined from absorbance data in Figure 2 and Beer's law from Figure 3 ) and the as-prepared concentration of the dispersions in NMP (solid curve) and the IPA (dashed curve). The $60 \mu \mathrm{g} / \mathrm{mL}$ dispersion was prepared by sonicating for 20 minutes and the subsequent dilutions from the $60 \mu \mathrm{g} / \mathrm{mL}$ dispersion were sonicated for 30 seconds each to homogenize. The dispersions were centrifuged after every successive dilution.

of sonication. Thus, the question arises whether the fraction of SWCNT removed during centrifugation consists only of nanotube aggregates. Therefore, the actual selectivity of the centrifugation process was examined further using the dispersions in NMP.

In a first experiment, a $2 \mu \mathrm{g} \mathrm{mL}^{-1}$ SWCNT dispersion in NMP was sonicated for 20 minutes and centrifuged twice. Figure 5 shows the optical spectra of the dispersions after sonication only and after the first and second centrifugations.

The absorption maximum centered at $1030 \mathrm{~nm}$ continues to decrease with successive centrifugation. The value of $A_{1030}$ decreased by 0.008 a.u. after the first centrifugation and further by 0.009 a.u. after the second centrifugation, which is 3-4 times larger than the random error in the absorbance measurements (0.0024 a.u.). This means that either the first centrifugation did not completely remove the SWCNT aggregates present in the dispersion, which were then removed during the second centrifugation, or that a fraction of the individually suspended nanotubes is always removed during each centrifugation step in addition to the aggregates (if present). The former explanation is analogous to the results obtained by Shim et al. [41], who had employed multiple cycles of centrifugation to selectively remove amorphous carbon from the as-grown nanotube soot. They observed that the first centrifugation step did not remove all the amorphous carbon from the nanotube soot and that multiple cycles of centrifugation were required. It should be pointed out that the major motivation behind the study of Shim et al. [41] was to use centrifugation as a purification technique to remove amorphous carbon. The selectivity of centrifugation in removing the SWCNT aggregates with respect to

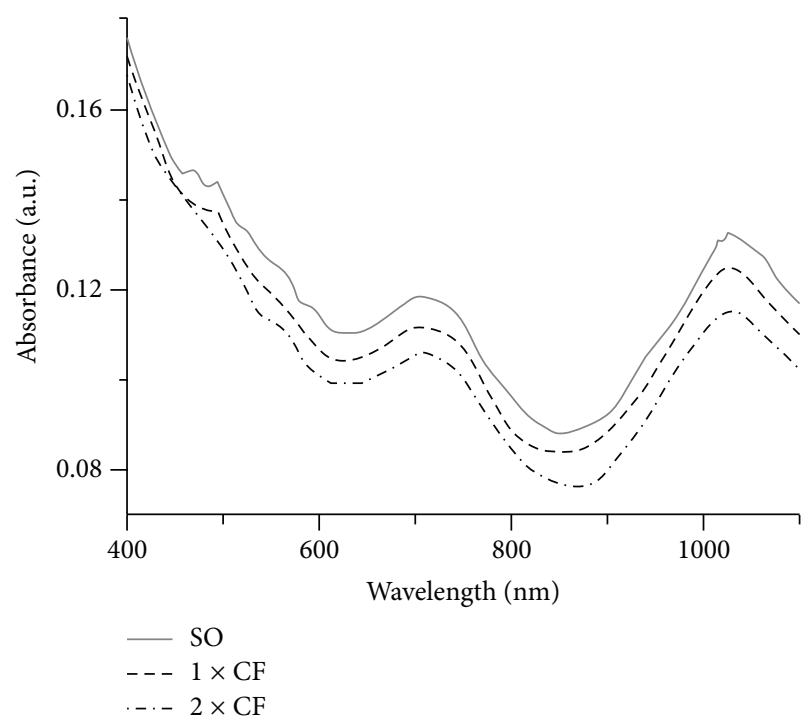

FIGURE 5: Absorption spectra of $2 \mu \mathrm{g} / \mathrm{mL}$ SWCNT dispersion in NMP after 20 minutes of sonication (solid curve) and after subsequent centrifugation (dashed curve) and recentrifugation with the same parameters (dashed-dotted curve). The following values of $A_{1030}$ were measured after sonication and first and second centrifugation, respectively: $A_{1030-\mathrm{so}}=0.132$ a.u., $A_{1030-1 \times \mathrm{CF}}=0.124$ a.u., and $A_{1030-2 x \mathrm{CF}}=0.115$ a.u.

the dispersed nanotubes was not studied by the authors [41]. The consequences of the two possible explanations in estimating the aggregation fraction will be dealt with in more detail in the next section.

3.4.2. Aggregation Factor: Effects of Sonication and Centrifugation. In the following experiment, dispersions were prepared with a part of the SWCNTs left intentionally as aggregates by sonicating them for lesser duration. A series of $7.5 \mu \mathrm{g} \mathrm{mL}^{-1}$ SWCNT dispersions (50 mL each) in NMP were sonicated for a range of durations between 3 and 30 minutes (corresponding to 0.05 and $0.5 \mathrm{~kJ} / \mathrm{mL}$ of sonication energy per unit volume). For the dispersions sonicated for less than 10 minutes, fine suspended SWCNT particulates could be observed visually, while samples sonicated for longer durations resulted in clear dispersions. The measured absorption should be affected by the presence of the aggregates. The difference between this absorbance compared to that of the dispersions with dispersed nanotubes (with long sonication durations) can then be used for calculating the true aggregation factor $\left(\eta_{\text {agg }}\right)$. The particular concentration of $7.5 \mu \mathrm{g} \mathrm{mL} \mathrm{L}^{-1}$ was chosen as it is below the dispersion limit of NMP [33] and any aggregation present would be a result of the processing conditions of the dispersions and not of solvent saturation effects. Typical absorbance spectra for dispersions sonicated for three different sonication times are shown in Figure 6.

No considerable difference between the spectra obtained from the dispersions sonicated for 20 and 30 minutes (curves i and iii in Figure 6) was observed. The initial absorbance (after sonication only) of the dispersion sonicated for 


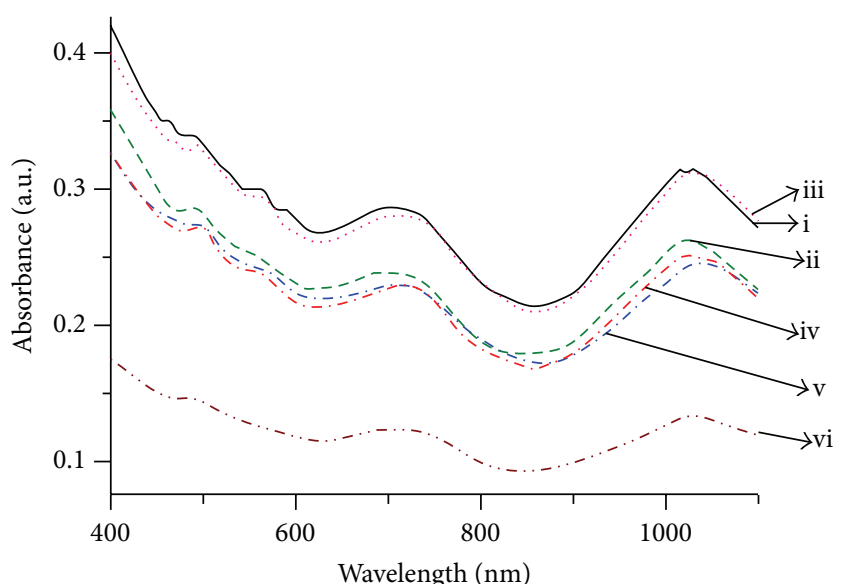
(i) 30 min_SO
-.. (iv) 20 min_CF
(ii) 30 min_CF
-.. (v) 4 min_SO
(iii) 20 min_SO
-... (vi) 4 min_CF

Figure 6: Optical spectra of $7.5 \mu \mathrm{g} / \mathrm{mL}$ SWCNT dispersions in NMP sonicated for 30 minutes ((i) and (ii)), 20 minutes ((iii) and (iv)), and 4 minutes ((v) and (vi)) before ((i), (iii), and (v)) and after centrifugation ((ii), (iv), and (vi)).

4 minutes is much lower than the ones sonicated for longer duration. This shows the influence of the nanotube aggregates (shielding effect) in the absorbance spectra of dispersions having large aggregates. Knowing $\varepsilon_{1030}$ (from Figure 3), the "apparent" concentration of the various dispersions was determined from the absorption data in Figure 6 using Beer's law (Figure 7).

In the case of sonication only (Figure 7), the calculated apparent concentration of the dispersions varied from 6.4 to $8 \mu \mathrm{g} \mathrm{mL} \mathrm{L}^{-1}$ depending on the sonication duration with no considerable change in the concentration after 10 minutes of sonication. After 20 minutes of sonication, the effective concentration reached a value of $8 \mu \mathrm{g} \mathrm{mL}^{-1}$, which could be regarded as the target concentration. Importantly, no significant change in the measured absorbance was observed indicating that the dispersions sonicated for 20-30 minutes or more can be considered aggregate-free. The absorbance data (curves $i$ and ii in Figure 6), however, show that after centrifugation, there is still a decrease in the effective concentration of the (aggregate-free) dispersion sonicated for 30 minutes. This might support our earlier hypothesis that centrifugation, unless optimized well, leads to the removal of a fraction of dispersed nanotubes (individual or narrow-bundles).

3.4.3. True Aggregation Factor. The SWCNT dispersion in NMP sonicated for 30 minutes (before centrifugation) has been shown to be practically aggregates-free and hence can be considered as the reference dispersion. So, the absorbance contribution from the reference dispersion $\left(A_{\lambda, \mathrm{SO}_{3} 30}\right)$ is a function of the total SWCNT concentration and the aggregation factor, $\eta_{\text {agg }}$, will therefore be defined with respect to this. When the dispersions sonicated for lesser than 30 minutes are centrifuged, the precipitate contains a combination of the SWCNT aggregates along with a fraction of dispersed

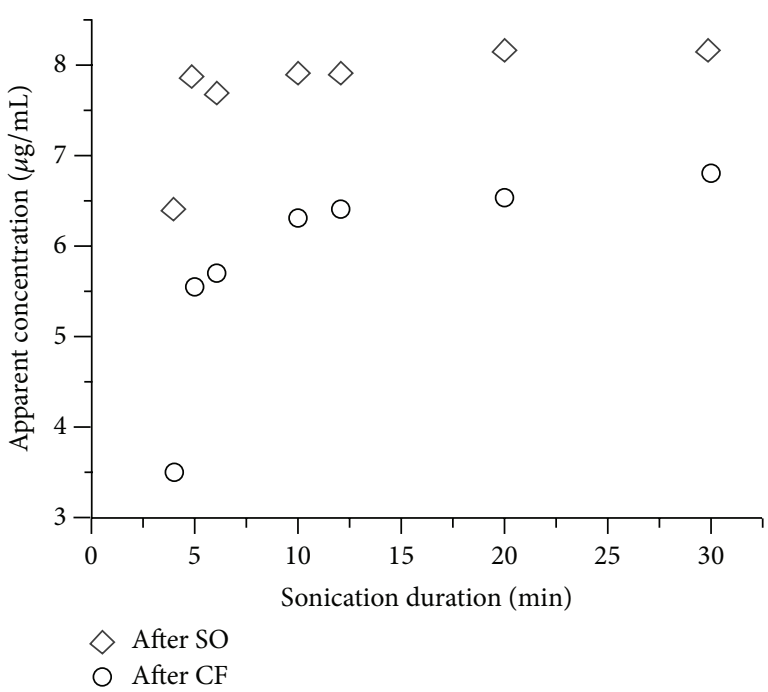

FIGURE 7: Change in the apparent concentration of NMP dispersions for different sonication durations (as determined from Beer's law and Figure 3 ) after sonication and after sonication-centrifugation.

nanotubes (individual or narrow-bundles), as established in the previous section. This is represented by the absorbance difference $\left[A_{\lambda, \mathrm{SO}_{3} 30}-A_{\lambda, \mathrm{CF} t}\right]$ (for $t<30$ ). For estimating the true aggregation factor, the absorbance contribution of the dispersed nanotubes removed during centrifugation has to be subtracted from $\left[A_{\lambda, \mathrm{SO}_{3} 30}-A_{\lambda, \mathrm{CF}_{-}}\right]$. The term $\left[A_{\lambda, \mathrm{SO}_{3} 30}-A_{\lambda, \mathrm{CF}_{3} 30}\right]$ represents this fraction of dispersed nanotubes removed unintentionally during centrifugation and has been incorporated as a correction factor to calculate $\eta_{\text {agg }}$. Hence, $\left[A_{\lambda, \mathrm{SO}_{3} 30}-A_{\lambda, \mathrm{CF}_{t}}\right]-\left[A_{\lambda, \mathrm{SO}_{3} 30}-A_{\lambda, \mathrm{CF}_{-30}}\right]$, which is proportional to the true fraction of SWCNT aggregates in the dispersion, divided by $\left(A_{\lambda, \mathrm{SO}_{3} 30}\right)$, which is proportional to the total SWCNT concentration, is therefore defined as the true aggregation factor $\left(\eta_{\text {agg }}\right)$ :

$$
\eta_{\mathrm{agg}}=\frac{\left[A_{\lambda, \mathrm{SO} \_30}-A_{\lambda, \mathrm{CF}-t}\right]-\left[A_{\lambda, \mathrm{SO} \__{-} 30}-A_{\lambda, \mathrm{CF} \_30}\right]}{A_{\lambda, \mathrm{SO} \_30}},
$$

which can be further simplified to

$$
\eta_{\text {agg }}=\frac{A_{\lambda, \mathrm{CF} \_30}-A_{\lambda, \mathrm{CF}\lrcorner} .}{A_{\lambda, \mathrm{SO} \_30}} .
$$

Equation (3) can therefore be used to calculate the aggregation factor of nanotubes in SWCNT dispersions sonicated for $t<30$ minutes (for the sonication power used in our experiments).

The change of $\eta_{\text {agg }}$ with sonication energy is shown in Figure 8 .

The aggregation factor decreases quickly with increasing sonication time and does not change appreciably beyond 12 minutes of sonication. For the SWCNTs used in this study with NMP as the dispersion solvent, the optimal sonication duration is therefore found to be around 12 minutes. This followed by a centrifugation step results in dispersions 


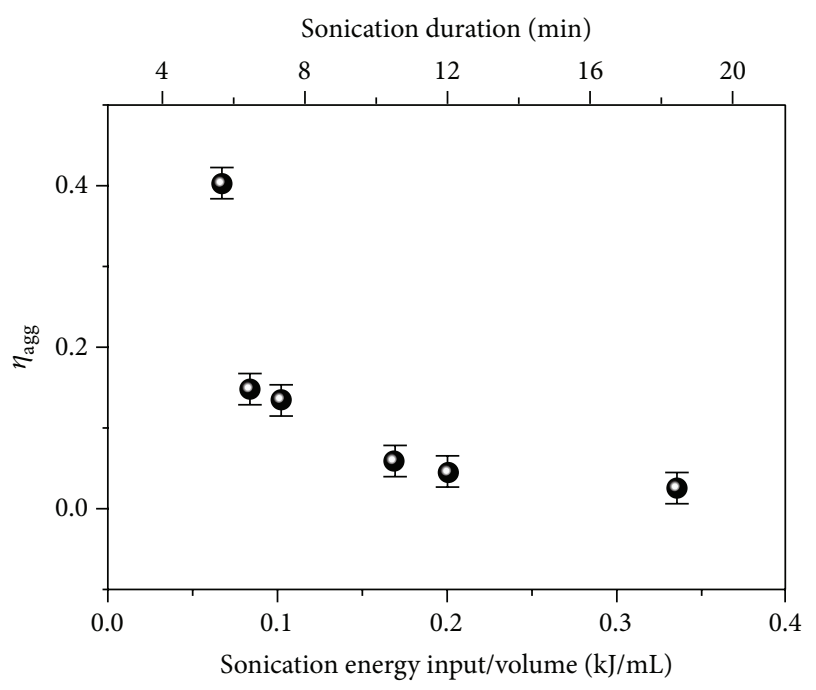

FIGURE 8: The aggregation factor $\left(\eta_{\mathrm{agg}}\right)$ as a function of sonication time and energy input per unit volume $(50 \mathrm{~mL}$ of each dispersion was sonicated). From the random error in the absorbance measurements ( 0.0024 a.u.), the propagated error in $\eta_{\text {agg }}$ was calculated to be 0.019 .

with a very low aggregation fraction of about 0.02 . Further experiments to study the change in the $I_{\mathrm{D}} / I_{\mathrm{G}}$ ratio and $\eta_{\text {agg }}$ with different sonication energy inputs for a given sonication duration (by changing the sonication power) and vice versa could be performed. Such studies will be further useful to determine the importance of sonication time and energy input relative to each other to minimize nanotube damage during sonication. It is worth noting that Figure 8 has been plotted for a specific target concentration of $7.5 \mu \mathrm{g} \mathrm{mL}^{-1}$. $\eta_{\text {agg }}$ could, however, be dependent on the dispersion concentration, even though it has been defined as a dimensionless parameter. The concentration dependence of $\eta_{\text {agg }}$ could be established by measuring the effect of sonication time on the apparent concentration for different target concentrations.

3.4.4. Red Shift of Absorbance Peak Maxima. Naumov et al. reported [37] that the presence of aggregates in the SWCNT dispersions causes a red shift (change towards higher wavelengths) in the maxima of the absorption peaks. Figure 9 shows the red shift of the absorption maxima as a function of the sonication duration.

We clearly see a red shift in the absorption maxima of the S22 peaks with decreasing sonication duration (increasing aggregation factor). The wavelength of the absorption maximum increases from $1029 \mathrm{~nm}$ for sonication duration of 30 minutes $\left(\eta_{\text {agg }}<0.02\right)$ to $1040 \mathrm{~nm}$ at 3 minutes of sonication $\left(\eta_{\text {agg }}=0.4\right)$. This red shift in absorption peak of SWCNTs with increasing levels of aggregation has been attributed to possible electronic coupling effects of nanotube bundles [37].

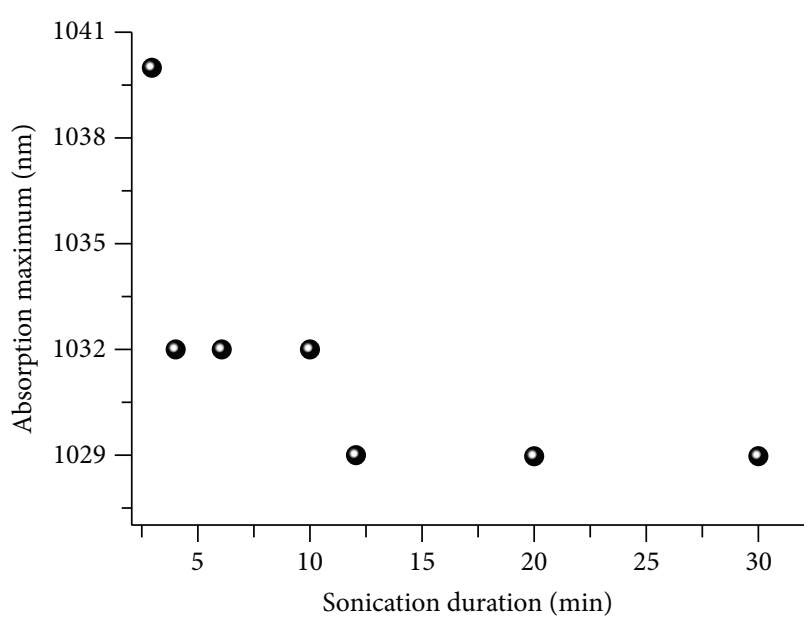

FIGURE 9: Red shift of the absorption maxima of the $S 22$ peaks (from the data in Figure 2) in the optical spectra of the dispersions in NMP with decreasing sonication duration (increasing aggregation factor).

\subsection{Dielectrophoresis with SWCNTs}

3.5.1. Effect of SWCNT Aggregation in Dielectrophoresis. Directed assembly of SWCNTs onto prefabricated electrode structures on a Si wafer was carried out by dielectrophoresis (DEP) [42]. Figure 10 shows typical scanning electron microscopy (SEM) images of electrode pairs with successful SWCNT assembly. The spatial resolution of SEM is not high enough to distinguish a single SWCNT from a narrow bundle of nanotubes. Atomic force microscopy (AFM) is often used to distinguish between a single SWCNT and nanotube bundles $[32,33,38,40]$. In our experiments, the SWCNTs were suspended above the underlying oxide layer between the electrode pairs and hence it was difficult to image them with AFM. Since AFM could not be used to image the suspended nanotubes between the electrode pairs, further evidence to prove the presence of individually dispersed nanotubes in the dispersions will be provided in the following section with the help of electromechanical measurements and modeling.

Detailed results on the dielectrophoresis of SWCNTs will be published in another report [43]. In short, some electrode pairs were bridged by a single (or a narrow-bundle) SWNCT (Figure 10(a)) and some by SWCNT aggregate(s) (Figure 10(b)). These results qualitatively confirm the presence of dispersed SWCNTs (individual or narrow bundles) in the NMP dispersions used during DEP (Figure 10(a)).

3.5.2. Electromechanical Measurements. After DEP, electromechanical measurements were performed with selected devices comprising of suspended nanotubes (single or narrow bundle?) deposited between the electrode pairs, like the device shown in Figure 10(a). Figure 11(a) shows the schematics of the electrical setup used to measure the mechanical resonance frequency of an oscillating nanotube under the influence of an external time-dependent electric field. The resistance of the nanotube was calculated from the voltage drop (measured across the two electrodes), normalized, 


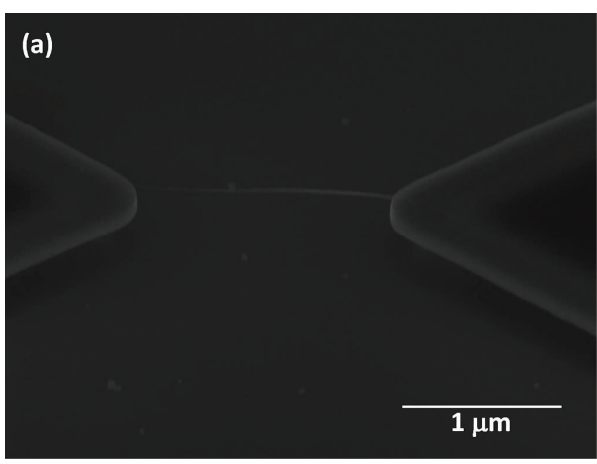

(a)

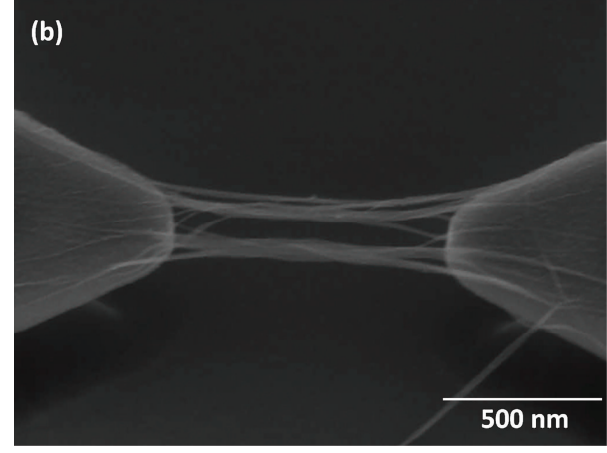

(b)

FIGURE 10: Scanning electron microscope images of (a) a single suspended SWCNT and (b) suspended SWCNT aggregates bridging an electrode pair after dielectrophoresis.

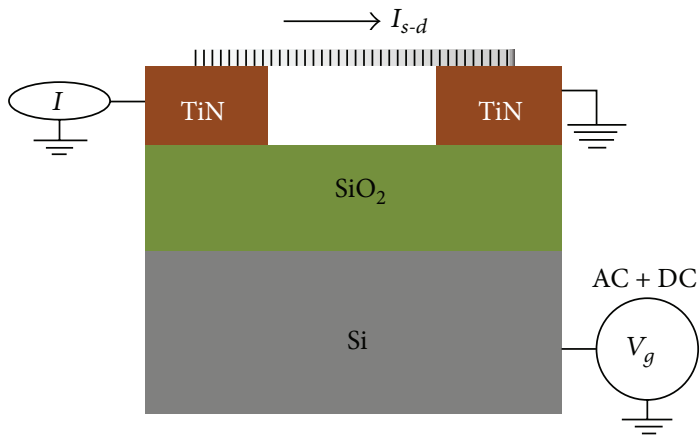

(a)

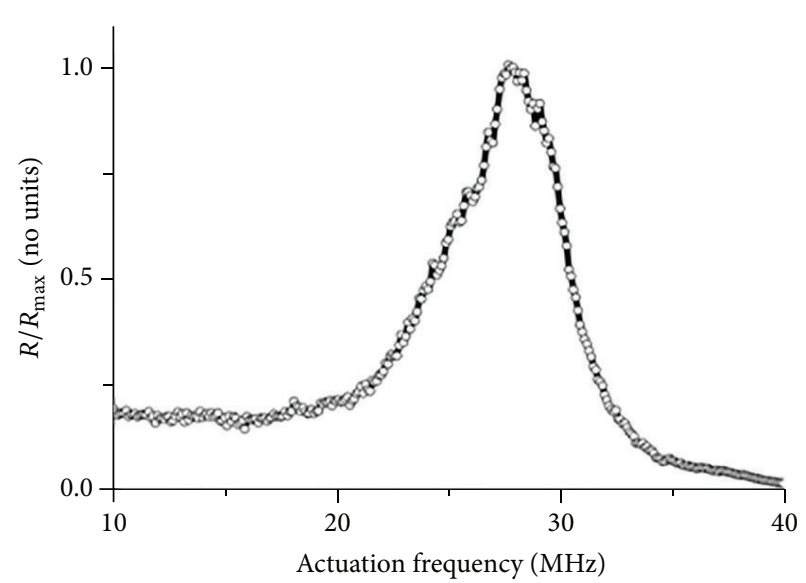

(b)

FIGURE 11: (a) Schematics of the resonance frequency measurement of the suspended nanotube resonator. A constant current was passed through the nanotube, while the actuation frequency applied at the substrate was swept from $10 \mathrm{kHz}$ to $50 \mathrm{MHz}$. The resistance change during the frequency sweep was calculated from the voltage drop across the two contact electrodes bridged by the nanotube. (b) A plot of timeaveraged normalized resistance of a typical suspended nanotube device as a function of the actuation frequency. A constant current of $20 \mathrm{nA}$ was passed through the nanotube, while an AC signal with an RMS amplitude of $1 \mathrm{~V}$ was applied at the substrate with a DC offset of $10 \mathrm{~V}$.

and then plotted as a function of the actuation frequency applied at the Si substrate (Figure 11(b)).

A detailed report on the results of the electromechanical measurements and modeling of the results is being considered for publication elsewhere [44]. In short, we note that the normalized resistance of the SWCNTs changes significantly at a particular frequency, leading to a peak in the resistance versus frequency plot. In Figure 11(b), the resistance maximum is measured at an actuation frequency corresponding to $27.5 \mathrm{MHz}$. This frequency, at which the resistance is maximum, has been shown to correspond to the mechanical resonance frequency of the suspended structure [45]. Furthermore, it has been demonstrated in our report [44] that the resonating nanotube can be modeled as an oscillating spring. Using the spring model, the resonance frequency of the fundamental flexural mode resonance frequency of a typical individual SWCNT was calculated to be $24.5 \mathrm{MHz}$. This shows a good agreement between the resonance frequency measured experimentally and the value calculated from the model of a single SWCNT resonator. It was also found that the number of electrode pairs bridged with SWCNT aggregates decreased with SWCNT concentration, as expected. Also, the dispersions that were centrifuged resulted in almost a twofold increase in the number of electrode pairs with an individual SWCNT assembly, compared to the dispersion of same concentration without centrifugation [44]. Hence, the results from the dielectrophoresis experiments and the electromechanical measurements validate the presence of individually dispersed nanotubes in our dispersions.

\section{Conclusions}

We have quantified the aggregation factor of SWCNTs in liquid media using optical absorption spectroscopy. This was performed through a series of controlled experiments involving dispersions of different concentrations, known sonication 


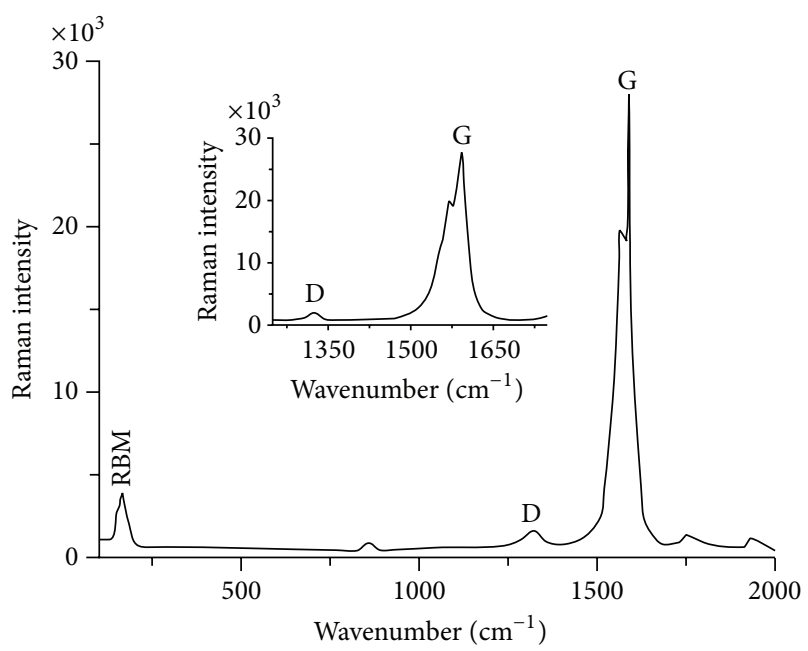

(a)

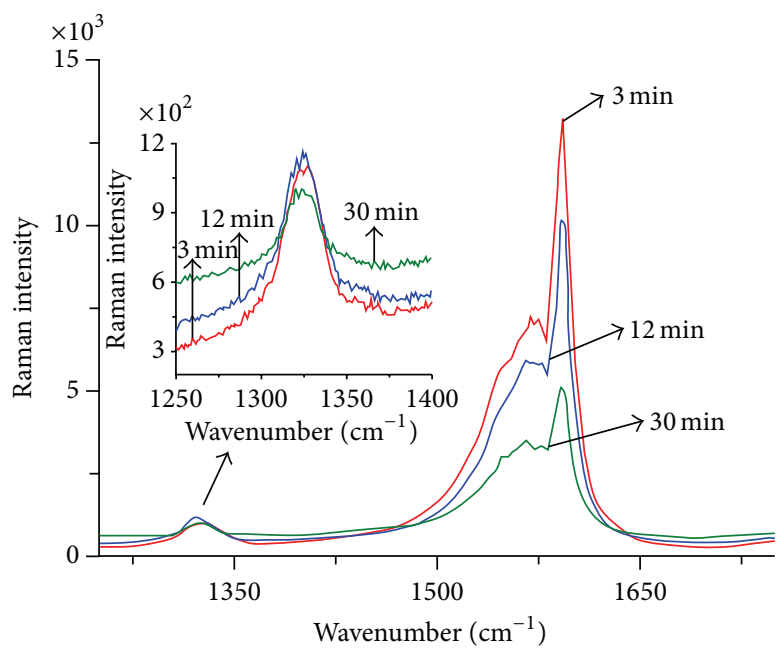

(b)

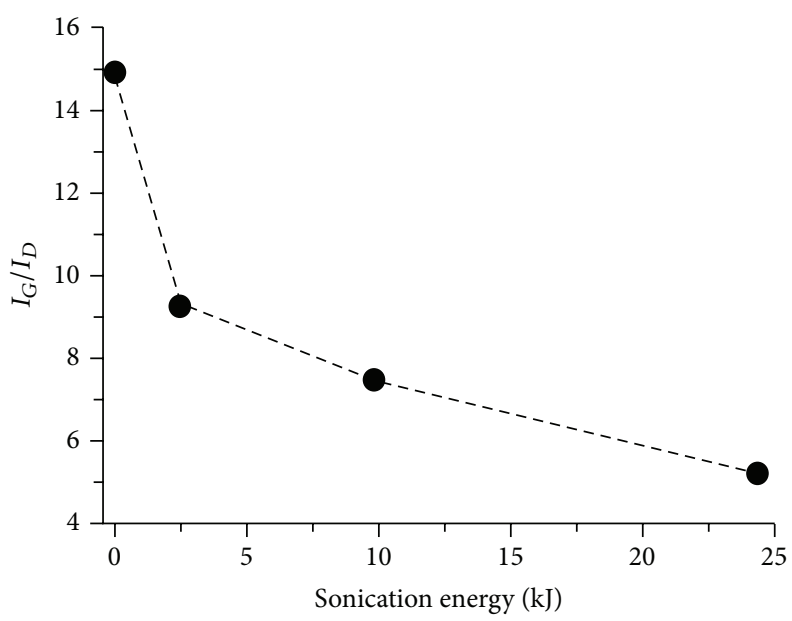

(c)

FIGURE 12: (a) Raman spectrum of the as-received SWCNT thin film with the RBM at $164 \mathrm{~cm}^{-1}$ and G and D peaks at $1590 \mathrm{~cm}^{-1}$ and $1322 \mathrm{~cm}^{-1}$, respectively. Inset: detailed view of the G and the D peaks of the as-received SWCNT thin film. (b) The G and D peaks of the drop-casted SWCNTs from the $60 \mu \mathrm{g} / \mathrm{mL}$ NMP dispersions that were sonicated for 3 minutes (red curve), 12 minutes (blue curve), and 30 minutes (green curve). Inset: detailed view of the D peaks. (c) The decrease in the $I_{\mathrm{G}} / I_{\mathrm{D}}$ ratio of the SWCNTs from the $60 \mu \mathrm{g} / \mathrm{mL}$ NMP dispersions with increasing sonication energy input. The $I_{\mathrm{G}} / I_{\mathrm{D}}$ ratio of the as-received SWCNT sample is plotted at zero sonication energy. The $I_{\mathrm{G}} / I_{\mathrm{D}}$ ratio was determined by measuring the peak heights of the $\mathrm{G}$ and the $\mathrm{D}$ peaks, respectively, with respect to the baseline.

energy input, and centrifugation steps with NMP and IPA as dispersion solvents. The importance of understanding the true effects of centrifugation has been pointed out for estimating the aggregation factor of SWCNTs. In cases where centrifugation also removes a part of the dispersed nanotubes in addition to the aggregates, the expression commonly used in the literature for calculating the fraction of SWCNT aggregates needs to be used with prudence. A modified approach to estimate the SWCNT aggregation factor in dispersions was proposed and verified.

Also, the net sonication energy input to exfoliate nanotubes has been calculated by calibrating the sonicator. The role of NMP as an effective solvent for dispersing SWCNTs has been reaffirmed with our data. NMP could be used to prepare uniform dispersions over a wide concentration range with minimal levels of aggregation. No considerable nanotube reaggregation was observed over a period of at least 35 days in the absence of additional processing. The SWCNT dispersions prepared from NMP were successfully used for directed assembly of the nanotubes by DEP on prefabricated electrode structures. The SEM results from the dielectrophoresis experiments and the post-DEP electromechanical measurements validate the presence of individually dispersed nanotubes in the dispersions. Optical spectroscopy could gain importance as an effective characterization tool for SWCNT device fabrication schemes like dielectrophoresis, where it is essential to have aggregates-free dispersions to enable controlled on-chip assembly of SWCNTs. 


\section{Conflict of Interests}

The authors declare that there is no conflict of interests regarding the publication of this paper.

\section{References}

[1] P. L. McEuen, "Single-wall carbon nanotubes," Physics World, vol. 13, no. 6, pp. 31-36, 2000.

[2] J. Kong, C. Zhou, A. Morpurgo et al., "Synthesis, integration, and electrical properties of individual single-walled carbon nanotubes," Applied Physics A: Materials Science and Processing, vol. 69, no. 3, pp. 305-308, 1999.

[3] C. Zhou, J. Kong, and H. Dai, "Intrinsic electrical properties of individual single-walled carbon nanotubes with small band gaps," Physical Review Letters, vol. 84, no. 24, pp. 5604-5607, 2000.

[4] J. Salvetat, G. A. D. Briggs, J. Bonard et al., "Elastic and shear moduli of single-walled carbon nanotube ropes," Physical Review Letters, vol. 82, no. 5, pp. 944-947, 1999.

[5] T. I. Jeon, K. J. Kim, C. Kang et al., "Optical and electrical properties of preferentially anisotropic single-walled carbonnanotube films in terahertz region," Journal of Applied Physics, vol. 95, no. 10, pp. 5736-5740, 2004.

[6] J. Hone, M. Whitney, C. Piskoti, and A. Zettl, "Thermal conductivity of single-walled carbon nanotubes," Physical Review B-Condensed Matter and Materials Physics, vol. 59, no. 4, pp. R2514-R2516, 1999.

[7] C. T. White and J. W. Mintmire, "Fundamental properties of single-wall carbon nanotubes," Journal of Physical Chemistry B, vol. 109, no. 1, pp. 52-65, 2005.

[8] N. Sinha, J. Ma, and J. T. W. Yeow, "Carbon nanotube-based sensors," Journal of Nanoscience and Nanotechnology, vol. 6, no. 3, pp. 573-590, 2006.

[9] A. Star, V. Joshi, S. Skarupo, D. Thomas, and J. P. Gabriel, "Gas sensor array based on metal-decorated carbon nanotubes," Journal of Physical Chemistry B, vol. 110, no. 42, pp. 21014-21020, 2006.

[10] Ph. Avouris, "Molecular electronics with carbon nanotubes," Accounts of Chemical Research, vol. 35, no. 12, pp. 1026-1034, 2002.

[11] J. Robertson, G. Zhong, H. Telg et al., "Carbon nanotubes for interconnects in VLSI integrated circuits," Physica Status Solidi (B) Basic Research, vol. 245, no. 10, pp. 2303-2307, 2008.

[12] V. Sazonova, Y. Yalsh, I. Üstünel, D. Roundy, T. A. Arlas, and P. L. McEuen, "A tunable carbon nanotube electrochemical oscillator," Nature, vol. 431, no. 7006, pp. 284-287, 2004.

[13] N. D. Jonge, M. Allioux, M. Doytcheva et al., "Characterization of the field emission properties of individual thin carbon nanotubes," Applied Physics Letters, vol. 85, no. 9, pp. 1607-1609, 2004.

[14] L. Ci, J. Suhr, V. Pushparaj, X. Zhang, and P. M. Ajayan, "Continuous carbon nanotube reinforced composites," Nano Letters, vol. 8, no. 9, pp. 2762-2766, 2008.

[15] A. A. Bhirde, V. Patel, J. Gavard et al., "Targeted killing of cancer cells in vivo and in vitro with EGF-directed carbon nanotubebased drug delivery," ACS Nano, vol. 3, no. 2, pp. 307-316, 2009.

[16] H. Dai, "Carbon nanotubes: synthesis, integration, and properties," Accounts of Chemical Research, vol. 35, no. 12, pp. 10351044, 2002.
[17] X. Li, X. Tu, S. Zaric et al., "Selective synthesis combined with chemical separation of single-walled carbon nanotubes for chirality selection," Journal of the American Chemical Society, vol. 129, no. 51, pp. 15770-15771, 2007.

[18] S. Pisana, A. Jungen, C. Zhang et al., "Flying and crawling modes during surface-bound single wall carbon nanotube growth," Journal of Physical Chemistry C, vol. 111, no. 46, pp. 17249-17253, 2007.

[19] Y. Li, S. Peng, D. Mann et al., "On the origin of preferential growth of semiconducting single-walled carbon nanotubes," Journal of Physical Chemistry B, vol. 109, no. 15, pp. 6968-6971, 2005.

[20] S. Ghosh, S. M. Bachilo, and R. B. Weisman, "Advanced sorting of single-walled carbon nanotubes by nonlinear densitygradient ultracentrifugation," Nature Nanotechnology, vol. 5, no. 6, pp. 443-450, 2010.

[21] F. Hennrich, S. Lebedkin, and M. M. Kappes, "Improving separation techniques for single-walled carbon nanotubes: towards monodisperse samples," Physica Status Solidi (B) Basic Research, vol. 245, no. 10, pp. 1951-1953, 2008.

[22] A. V. Naumov, O. A. Kuznetsov, A. R. Harutyunyan et al., "Quantifying the semiconducting fraction in single-walled carbon nanotube samples through comparative atomic force and photoluminescence microscopies," Nano Letters, vol. 9, no. 9, pp. 3203-3208, 2009.

[23] V. A. Davis, A. N. G. Parra-Vasquez, M. J. Green et al., "True solutions of single-walled carbon nanotubes for assembly into macroscopic materials," Nature Nanotechnology, vol. 4, no. 12, pp. 830-834, 2009.

[24] P. Angelikopoulos, A. Gromov, A. Leen, O. Nerushev, H. Bock, and E. E. B. Campbell, "Dispersing individual single-wall carbon nanotubes in aqueous surfactant solutions below the cmc," Journal of Physical Chemistry C, vol. 114, no. 1, pp. 2-9, 2010.

[25] L. Vaisman, H. D. Wagner, and G. Marom, "The role of surfactants in dispersion of carbon nanotubes," Advances in Colloid and Interface Science, vol. 128-130, pp. 37-46, 2006.

[26] M. C. Hersam, "Progress towards monodisperse single-walled carbon nanotubes," Nature Nanotechnology, vol. 3, no. 7, pp. 387-394, 2008.

[27] J. L. Bahr, E. T. Mickelson, M. J. Bronikowski, R. E. Smalley, and J. M. Tour, "Dissolution of small diameter single-wall carbon nanotubes in organic solvents?" Chemical Communications, no. 2, pp. 193-194, 2001.

[28] C. A. Furtado, U. J. Kim, H. R. Gutierrez, L. Pan, E. C. Dickey, and P. C. Eklund, "Debundling and dissolution of single-walled carbon nanotubes in amide solvents," Journal of the American Chemical Society, vol. 126, no. 19, pp. 6095-6105, 2004.

[29] S. D. Bergin, V. Nicolosi, P. V. Streich et al., "Towards solutions of single-walled carbon nanotubes in common solvents," Advanced Materials, vol. 20, no. 10, pp. 1876-1881, 2008.

[30] Y. Hernandez, V. Nicolosi, M. Lotya et al., "High-yield production of graphene by liquid-phase exfoliation of graphite," Nature Nanotechnology, vol. 3, no. 9, pp. 563-568, 2008.

[31] S. D. Bergin, Z. Sun, P. Streich, J. Hamilton, and J. N. Coleman, "New solvents for nanotubes: approaching the dispersibility of surfactants," Journal of Physical Chemistry C, vol. 114, no. 1, pp. 231-237, 2010.

[32] S. Giordani, S. D. Bergin, V. Nicolosi et al., "Debundling of single-walled nanotubes by dilution: observation of large populations of individual nanotubes in amide solvent dispersions," 
Journal of Physical Chemistry B, vol. 110, no. 32, pp. 15708-15718, 2006.

[33] Q. Cheng, S. Debnath, L. O’Neill, T. G. Hedderman, E. Gregan, and H. J. Byrne, "Systematic study of the dispersion of swnts in organic solvents," Journal of Physical Chemistry C, vol. 114, no. 11, pp. 4857-4863, 2010.

[34] B. J. Landi, H. J. Ruf, J. J. Worman, and R. P. Raffaelle, "Effects of alkyl amide solvents on the dispersion of single-wall carbon nanotubes," Journal of Physical Chemistry B, vol. 108, no. 44, pp. 17089-17095, 2004.

[35] M. E. Itkis, D. E. Perea, R. Jung, S. Niyogi, and R. C. Haddon, "Comparison of analytical techniques for purity evaluation of single-walled carbon nanotubes," Journal of the American Chemical Society, vol. 127, no. 10, pp. 3439-3448, 2005.

[36] S. Ohmori, T. Saito, M. Tange et al., "Fundamental importance of background analysis in precise characterization of singlewalled carbon nanotubes by optical absorption spectroscopy," Journal of Physical Chemistry C, vol. 114, no. 22, pp. 10077-10081, 2010.

[37] A. V. Naumov, S. Ghosh, D. A. Tsyboulski, S. M. Bachilo, and R. B. Weisman, "Analyzing absorption backgrounds in singlewalled carbon nanotube spectra," ACS Nano, vol. 5, no. 3, pp. 1639-1648, 2011.

[38] P. Vichchulada, M. A. Cauble, E. A. Abdi, E. I. Obi, Q. Zhang, and M. D. Lay, "Sonication power for length control of singlewalled carbon nanotubes in aqueous suspensions used for 2dimensional network formation," Journal of Physical Chemistry C, vol. 114, no. 29, pp. 12490-12495, 2010.

[39] Q. Cheng, S. Debnath, E. Gregan, and H. J. Byrne, "Ultrasoundassisted SWNTs dispersion: effects of sonication parameters and solvent properties," Journal of Physical Chemistry C, vol. 114, no. 19, pp. 8821-8827, 2010.

[40] A. J. Blanch, C. E. Lenehan, and J. S. Quinton, “Optimizing surfactant concentrations for dispersion of single-walled carbon nanotubes in aqueous solution," Journal of Physical Chemistry B, vol. 114, no. 30, pp. 9805-9811, 2010.

[41] J. Shim, P. Vichchulada, Q. Zhang, and M. D. Lay, "Spectroscopic and scanning probe studies of a nondestructive purification method for SWNT suspensions," Journal of Physical Chemistry C, vol. 114, no. 1, pp. 652-657, 2010.

[42] A. Vijayaraghavan, S. Blatt, D. Weissenberger et al., "Ultralarge-scale directed assembly of single-walled carbon nanotube devices," Nano Letters, vol. 7, no. 6, pp. 1556-1560, 2007.

[43] H. Pathangi, G. Groeseneken, and A. Witvrouw, "Directed assembly of horizontal suspended carbon nanotubes for NEMS applications," Microelectronic Engineering, 2011.

[44] H. Pathangi, V. Cherman, A. Khaled, B. Soree, G. Groeseneken, and A. Witvrouw, "Towards CMOS-compatible single-walled carbon nanotube resonators," Microelectronic Engineering, vol. 107, pp. 219-222, 2013.

[45] A. R. Hall, M. R. Falvo, R. Superfine, and S. Washburn, "A self-sensing nanomechanical resonator built on a single-walled carbon nanotube," Nano Letters, vol. 8, no. 11, pp. 3746-3749, 2008. 

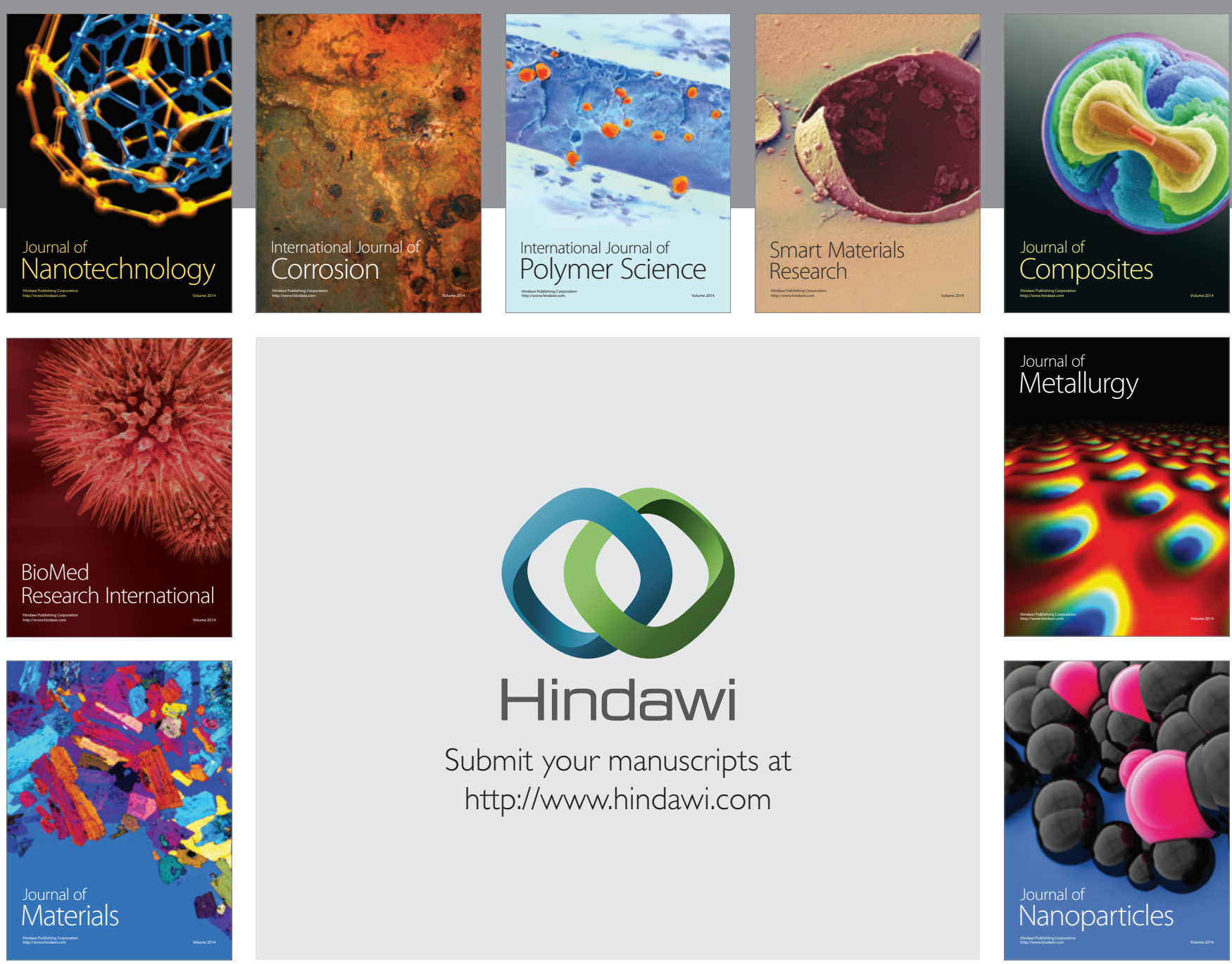

Submit your manuscripts at http://www.hindawi.com
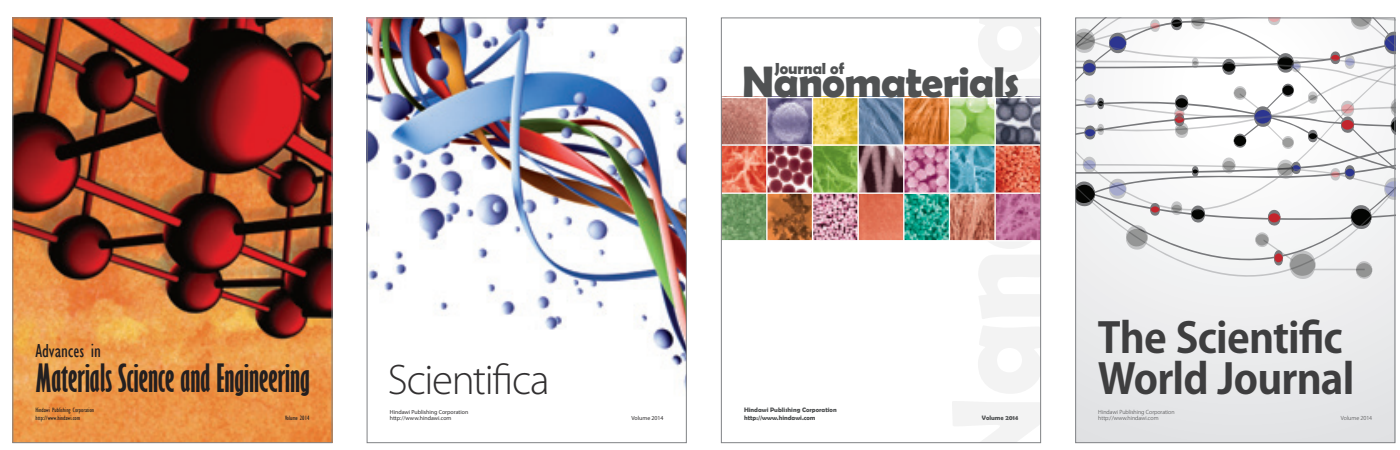

\section{The Scientific World Journal}
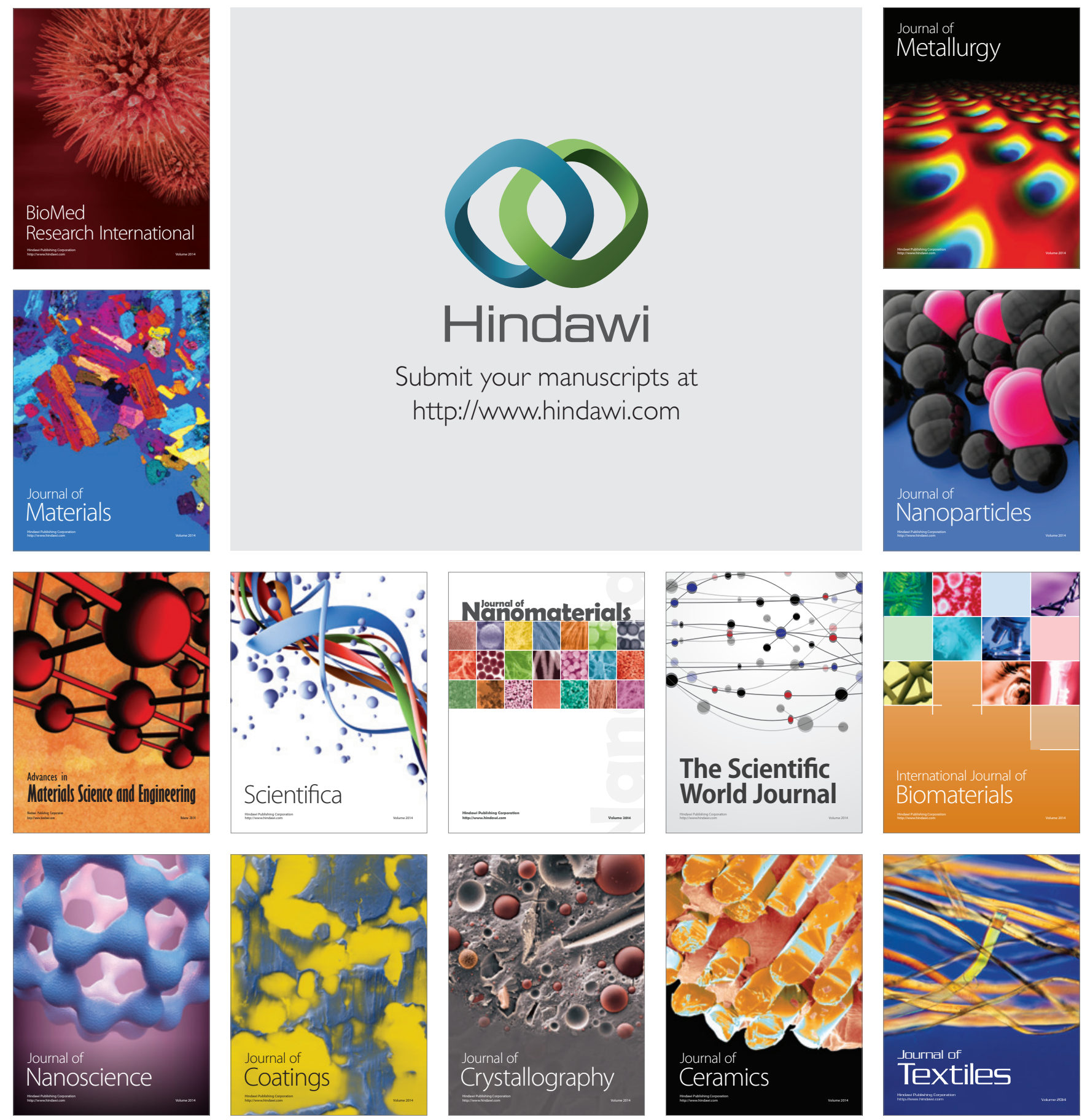